(2) Open Access Full Text Article

\title{
A self-reported questionnaire for quantifying illness symptoms in elite athletes
}

This article was published in the following Dove Press journal:

Open Access Journal of Sports Medicine

20 March 2010

Number of times this article has been viewed

\section{Alexander Matthews \\ David Pyne ${ }^{1,2}$ \\ Philo Saunders ${ }^{2}$ \\ Kieran Fallon ${ }^{1,2}$ \\ Peter Fricker ${ }^{1,2}$}

'Medical School, Australian National University, Canberra, ACT, Australia;

${ }^{2}$ Australian Institute of Sport,

Canberra, ACT, Australia
Correspondence: David Pyne Department of Physiology,Australian Institute of Sport, Belconnen, ACT, 2616, Australia

Email david.pyne@ausport.gov.au
Purpose: To develop and evaluate a questionnaire that quantifies the self-reported frequency, duration and severity of illness symptoms in highly-trained athletes. We examined whether runners had more symptoms than recreationally-active individuals, and whether runners more prone to illness were undertaking more strenuous training programs.

Methods: A daily illness questionnaire was administered for three months during the summer to quantify the type, frequency, duration, and severity of illness symptoms as well as the functional impact on the ability to undertake exercise performance. A total of 35 participants (12 highlytrained runners living in a community setting and 23 recreationally-active medical students) completed the questionnaire.

Results: Runners had a similar frequency of illness ( $2.1 \pm 1.2$ vs. $1.8 \pm 2.3$ episodes, mean $\pm \mathrm{SD}$, $P=0.58)$, but substantially longer duration $(5.5 \pm 9.9$ vs $2.8 \pm 3.1$ days, $P<0.01)$ and illness load ( $7.7 \pm 16.2$ vs $4.5 \pm 4.8$ units, $P=0.001)$ than age- and sex-matched recreationally-active individuals respectively. Runners more prone to illness symptoms had marginally higher training loads.

Conclusions: The athlete illness questionnaire is useful for quantifying the pattern of selfreported symptoms of illness in field settings. Highly-trained runners experience longer episodes of illness with a greater impact on daily activity than recreationally-active individuals.

Keywords: athlete, self-reported illness, questionnaire, exercise performance

\section{Introduction}

An important objective of highly-trained athletes is to remain healthy and perform consistently in training and competition. Periods of heavy training involving prolonged intense exercise have been associated with an increased incidence of upper respiratory tract illness (URTI). ${ }^{1}$ The timing of URTI is critical for competitive athletes. The pattern of URTI in athletes does not always follow the usual increased winter prevalence for the general population, and symptoms of illness are often reported during high intensity training, especially in the taper period leading up to competition. ${ }^{2}$ The period of days immediately following participation in competitive endurance sports has also been linked to an increased risk of illness in athletes. ${ }^{3}$ Mild illness appears not to adversely affect the primary physiological processes, but athletic performance may be compromised if moderate or more severe problems are encountered. ${ }^{4}$

Existing illness logs or questionnaires developed for the general community may not adequately meet the requirements of sports medicine practitioners, coaches and their athletes, and researchers in a sports medicine setting. Although some existing illness questionnaires incorporate a 'functional aspect', that is the degree to which subjects 
are impeded in carrying out activities (eg, sport, hobbies, and work, contributing to 'objective' symptom reporting), ${ }^{5}$ they are frequently disease-specific. This methodological approach does not directly meet the requirements of quantifying symptoms of illness in an athlete population, and a well-designed questionnaire that can be completed easily on a daily basis is required for clinical and research applications.

The Australian Institute of Sport Self-Reported Daily Illness Log is one instrument that has been used to investigate the pattern of illness symptoms in athletes. ${ }^{3}$ Differences in training intensity and volume were not associated with the incidence of respiratory illness in highly-trained distance runners, and symptoms associated with mild illness did not necessarily impair performance. ${ }^{3}$ The findings of this study, notwithstanding some minor methodological limitations, justify the development of an improved questionnaire for sports medicine applications. We recently reported a discrepancy between physician and laboratory-diagnosed infections in elite athletes, ${ }^{6}$ and an initial screening questionnaire provided by athletes could be a simple means of improving the diagnostic process.

Key limitations of existing questionnaires include the subjective nature and individual variability of symptom reporting and the reliability of self-reported measures of illness. In brief, the primary limitations are: a) length: questionnaires are either too long to complete on a daily basis, or too brief to include all the symptoms encountered by athletes; b) functionality: there may be no indication of the impact of symptoms on activity or performance, leading to subjectivity in self-reporting; d) specificity: the questionnaire may be too disease-specific to use for universal symptom-reporting in an athlete population or too sport-specific to use across a range of sports; e) layout: bias may be introduced by the chosen layout of 'symptom categories' (eg, presenting the most common symptoms at the beginning of the questionnaire may increase the reporting of these symptoms compared with those at the end); and f) format: a pen and paper format may not suit the current computer-literate athlete population. A computer-based questionnaire is easier to complete and facilitates rapid feedback and analysis for the athlete, coach, researcher, or physician.

A criticism of self-reported illness questionnaires is that symptom measures reflect subjectively experienced wellbeing rather than objective health status: that is, symptom reports do not necessarily reflect illness. ${ }^{7}$ One study used an illness behavior questionnaire (IBQ) in elite middle and long distance runners and questioned athlete self-reporting of illness resulting from 'subjective symptomatology'. ${ }^{8}$
Clearly athlete self-reporting of illness in a questionnaire may be at odds with objective assessment by a physician. Illness questionnaires can be improved by asking subjects to report symptoms in terms of the impact on their training or general activity, which may produce a more objective health record. We are optimistic that the incorporation of this feature will enhance the usefulness of a self-reported illness questionnaire.

Other illness questionnaires include the Wisconsin Upper Respiratory Symptom Survey which is specific for the common cold, ${ }^{9,10}$ the Lara Asthma Symptom Scale for asthma, and the St George's Respiratory Questionnaire ${ }^{11}$ for patients with chronic lung diseases such as chronic obstructive pulmonary disease. Each questionnaire includes 'functional' classifications of illness symptoms, although their specificity makes them largely inappropriate for illness reporting in athletes.

The illness perception questionnaire uses five scales: a) identity: patient symptoms; b) cause: personal ideas about etiology; c) timeline: duration of illness; d) consequences: effects and outcomes; and e) cure control: recovery. ${ }^{12}$ This questionnaire has an advantage in that it allows the user to add items to meet the needs of particular patient groups and is flexible for specific health threats. However, it is more appropriate for patients with chronic illnesses than as a daily self-reported illness instrument for athletes.

The direct impact of illness on athletic performance has been a topic of debate with little experimental investigation. Mild illness may have a small harmful mean effect on the performance of elite athletes, but there is a substantial chance for harmful effects on individual athletes. ${ }^{4}$ Moderate exercise can potentially improve immunity, but repetitive intense exercise in some athletes is immunosuppressive. ${ }^{13}$ Similarly, lower rates of illness have been observed in recreationally competitive athletes and substantially higher rates noted in highly-trained triathletes and cyclists, supporting the notion that heavy training loads may produce an increased incidence of illness. ${ }^{14}$ Given the high training loads of many athletes, these studies are informative and highlight the need for careful monitoring of the health of athletes. Some clinical guidelines have been developed for coaches to reduce the risk of illness that compromises training or competitive performance. ${ }^{15}$ A self-reported illness symptom questionnaire meeting current needs of athletes, coaches, physicians, and researchers will assist in diagnosing illness and managing training loads to reduce the incidence of illness.

The primary aim of this study was to develop a questionnaire that quantifies the pattern of illness symptoms in highly-trained 
athletes and characterize differences in type, frequency, duration, and severity of illness. A secondary aim was to use this questionnaire to compare the functional impact of symptoms on exercise performance and physical activity on highly-trained athletes and on recreationally-active individuals. We also sought to identify relationships between the training loads of competitive athletes and underlying patterns of illness.

\section{Methods}

\section{Subjects}

A total of 35 individuals participated in this study: 12 runners (9 male, 3 female) living in the broader community in Canberra, Australia (age $31.8 \pm 4.0 \mathrm{y}$, mean $\pm \mathrm{SD}$ ) and 22 Australian National University medical students ( 8 males, 15 females age $26.4 \pm 4.3 \mathrm{y}$ ).

\section{Recruitment}

Sports medicine physicians and scientists assisted with recruiting the group of 12 highly-trained athletes and the group of 22 medical students. Initial contact with the subjects was made in person and by email. Subjects were invited to participate and were free to withdraw at any time. The purpose of the questionnaire and the questions themselves were explained to all parties to ensure complete understanding and it was not blinded to the subjects. Written consent was obtained from each participant and this addressed privacy issues concerning access to personal information. The study was approved by the Ethics Committee of the Australian National University and the Australian Institute of Sport.

\section{Experimental design}

Two study groups (an experimental group of highly-trained athletes and a control group of age- and sex-matched recreationally-active individuals) were studied in a prospective observational controlled trial. We developed and implemented a questionnaire to determine whether illness symptoms have a direct impact on athletic/exercise performance (Figures 1,2). We sought to create an illness log that could be completed in no more than five minutes per day, was not specific to any disease-type, provided options that were clearly differentiated and easy for athletes to follow and address, and could be used by elite athletes in a broad range of sports.

\section{Questionnaire}

The self-reported illness questionnaire consisted of both a training diary and an illness diary for athletes to complete daily. The revised questionnaire was based on a version previously developed at the Australian Institute of Sport. ${ }^{3}$
Briefly, the questionnaire was revised following feedback from a group of medical experts; four from the Australian Institute of Sport and five from the Australian National University Medical School. The primary improvements to the original version included all the essential symptoms likely to be encountered by athletes, a layout designed with the intent to minimize bias in symptom reporting by athletes, and clearer directions for athletes to rate the severity of their symptoms. A particular focus of the interviews was direct questioning on features allowing 'functional' classification of illness in terms of direct impact on performance and how the illness questionnaire could be modified to enhance its utility in both research and clinical settings.

After the interview and information-gathering process, the final version of the questionnaire was produced in the format of a single sheet to capture all the details for each calendar month. Subjects were provided three copies of the questionnaire to cover the three months of the study period. Subjects were given verbal and written instructions on how to complete the questionnaire daily.

Subjects were required to record training intensity on a scale from 1-5 (rated 1 low; 5 high) and duration (minutes). From this data, training load (duration $\times$ intensity) was calculated and a weekly mean training load derived. Subjects recorded illness as (Yes/No), together with illness behavior (ie, doctor visits, blood tests, and medications) (Figure 1). When an illness was reported, subjects were required to identify the symptom(s) and the extent to which it (they) affected training from a list of symptom categories that included upper respiratory tract infection, chest infection, muscles and joints, gastrointestinal problems, and skin conditions (Table 1). Symptoms were recorded according to their type (category) and severity (minimal = normal training $[$ score $=1]$, moderate $=$ modified training $[$ score $=2]$, severe $=$ discontinued training $[$ score $=3]$ ). This differs from the terms (mild, moderate and severe) used in the original version of this questionnaire (12). We considered that 'minimal' was more suitable than 'mild' for a functional classification of illness (the effect of the symptom on exercise performance), as it would make athletes think about the effect of their symptoms on their training, and help them to report their symptoms in a more objective manner. The three level scale of functional impact was similar to methods used previously for assessment of fatigue in elite athletes undertaking prolonged physical activity. ${ }^{16}$

An illness was defined as a subject reporting one or more symptoms on two or more consecutive days, or when the 


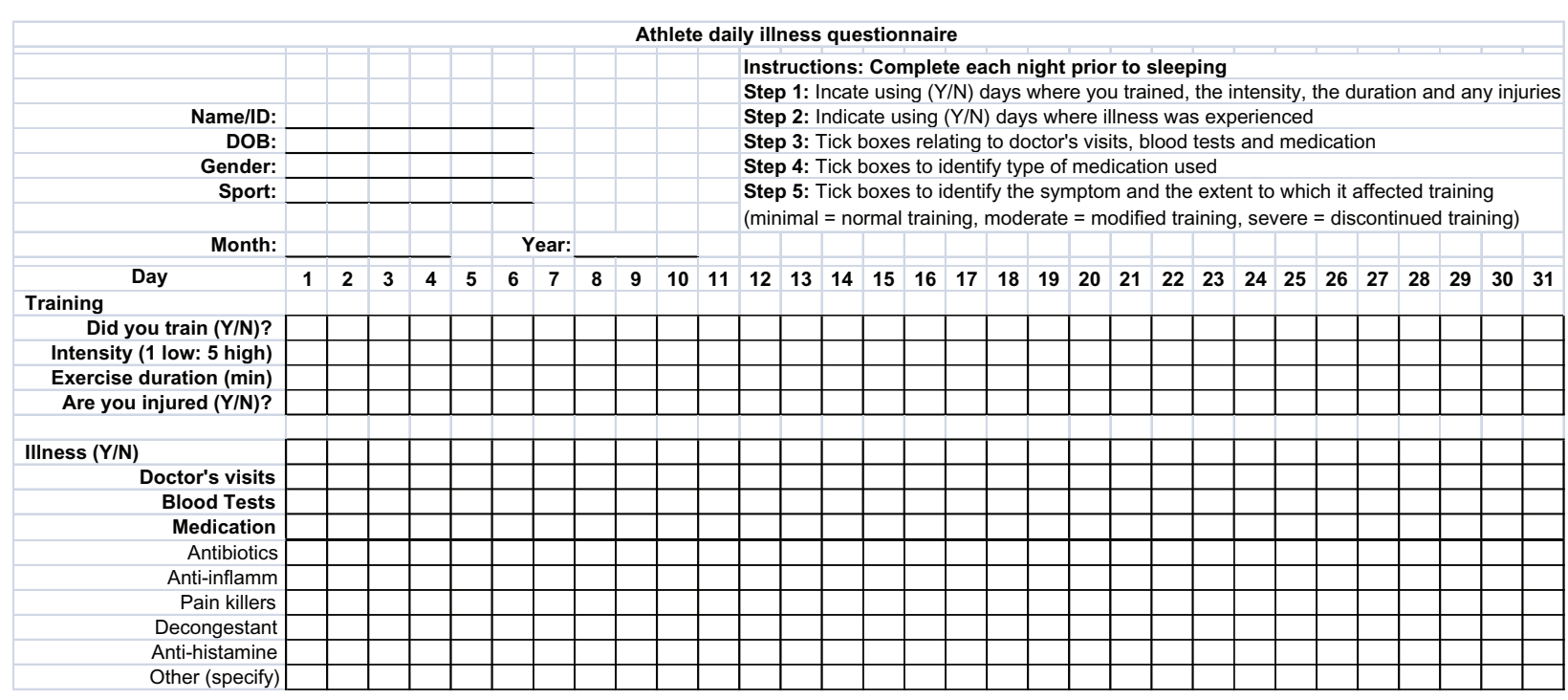

Figure I Top panel of the daily illness questionnaire showing demographic and personal details, the training duration and intensity (scaled low to high), the presence of illness, and details of medications.

severity was rated as either moderate or severe on one day. Symptoms separated by two days or less were regarded as a recurrence or continuation of the initial episode and were counted as part of the same episode. The following indices were calculated for each episode of illness: type, duration

Table I Athlete daily illness questionnaire: categories of symptoms
I. Upper respiratory - blocked or runny nose, sore throat, sneezing
2. Chest infection - coughing, sputum, chest congestion, wheezing, high temperature (give measurement)
3. Muscles and joints - aching or swollen (not related to injury)
4. Gastrointestinal problems - nausea, vomiting, diarrhea, abdominal pain, bloating, painful swallowing, loss of appetite
5. Head - headache, migraine, dizziness, vision impairment, vertigo, glare/light intolerance
6. Eye irritation - itchiness, redness, sticky discharge, watery eyes
7. Rashes - specify - localised (L) or widespread (W)
8. Skin infections - sores, boils, deep abscesses, infected blisters, athlete's foot
9. General fatigue - lethargy, tiredness
10. Cardiovascular - palpitations (sensation of heart beating @ rest), shortness of breath, blackouts
II. Ears - ear ache, ringing in the ears, hearing loss
12. Psychological - feeling depressed or anxious (not related to major event), poor sleeping pattern
13. Urinary tract - increased frequency of passing urine, pain/burning, bleeding, menstrual irregularity
14. Other (please specify)

Notes: The severity of a particular symptom on a given day was rated as either minimal (normal training), moderate (modified training) or severe (no training).A fully formatted version of the questionnaire is available from the corresponding author. (number of days), severity, and a total illness load. The total illness load was calculated as the cumulative sum of the daily severity scores for an illness episode, different to the method used previously where load was derived as the product of the total duration in days and peak severity. ${ }^{3}$ At the completion of the three month monitoring period, we invited the subjects to give verbal or written feedback on the questionnaire.

We also sought to determine whether highly-trained athletes have a greater frequency, duration or severity of illness symptoms than recreationally-active individuals (medical students). Runners were categorized as 'more prone' (three or more self-reported episodes of illness in the study period) or 'less prone' (two or fewer self-reported episodes of illness). We identified any substantial associations or trends related to the training loads of competitive athletes and underlying patterns of illness.

\section{Statistical analysis}

Descriptive statistics (mean, standard deviation, and coefficient of variation) were used to describe the patterns of illness between each study group. The frequency of illness (nominal level data) was root-transformed before analysis. The duration of illness (days) and the illness load were log-transformed before analysis to reduce bias arising from non-uniformity. The mean difference between athlete and recreationally-active groups and their $90 \%$ confidence limits were estimated via the unequal-variances $t$ statistic. The following criteria were employed to interpret the magnitude of standardized change or differences score: trivial $<0.2$, 
small $0.2-0.6$, medium $0.6-1.2$, large $1.2-2.0$, and very large $>2.0 .{ }^{17}$ The degree of between-subject variability in training load was indicated with the \% coefficient of variation. Significance was accepted at $P$-value $<0.05$.

\section{Results}

\section{Pattern of illness in subjects groups}

Table 2 shows the frequency, duration, and severity of self-reported symptoms of illness in runners and control subjects. Runners had a similar frequency of illness to recreationally-active individuals during the three month study period. However the typical duration of symptoms in runners ( $\sim 6$ days) was approximately twice that of the control subjects ( $\sim 3$ days). Similarly using the derived composite score of illness load (duration $\times$ severity), the runners experienced more substantial symptoms than the control subjects.

\section{Impact of training load on illness in runners}

Runners more prone to illness symptoms (three or more selfreported episodes of illness; $n=5$ ) had a marginally higher training load (229 \pm 106 arbitrary units, mean \pm standard deviation; $\%$ coefficient of variation $=46 \%$ ) than less prone runners (two or fewer episodes of illness; $n=7,202 \pm 61$ arbitrary units, $\%$ coefficient of variation $=30 \%)(P=0.63$, effect size $=0.34$, small $)$, but the imprecision of estimation precludes any firm conclusions on this relationship.

\section{Distribution of symptoms}

Table 3 shows the distribution of symptoms in both the runners and control subjects. The three most frequent self-reported symptoms were upper respiratory tract illness (URTI), headache and fatigue. Muscle and joint pain, and gastrointestinal symptoms were also reported. Rashes,

Table 2 Self-reported frequency, duration and index of illness load (arbitrary units) in highly-trained athletes and controls (recreationally-active medical students) over a three month summer training period (mean \pm standard deviation)

\begin{tabular}{llll}
\hline & $\begin{array}{l}\text { Frequency } \\
(\mathbf{n})\end{array}$ & $\begin{array}{l}\text { Duration } \\
\text { (days) }\end{array}$ & $\begin{array}{l}\text { Illness load } \\
\text { (duration } \times \\
\text { severity) }\end{array}$ \\
\hline Runners & $2.1 \pm 1.2$ & $5.5 \pm 9.9$ & $7.7 \pm 16.2$ \\
Controls & $1.8 \pm 2.3$ & $2.8 \pm 3.1$ & $4.5 \pm 4.8$ \\
$P$-value & 0.58 & 0.00001 & 0.001 \\
Effect size & $0.17 \pm 0.53$ & $0.74 \pm 0.27$ & $0.56 \pm 0.27$ \\
(Descriptor) & Trivial & Moderate & Small \\
\hline
\end{tabular}

cardiovascular problems, and urinary problems were not reported in either group during the three month study period.

\section{Participant feedback}

The following suggestions were made by athletes participating in our study to improve the self-reported illness questionnaire: 1) Injury: subjects noted that it is difficult to judge 'injury' via Yes/No criteria, as an athlete can have minor symptoms of discomfort which limit training, but not so uncomfortable that training is impossible. These symptoms might best be represented by a 'No, Low, Moderate, High' severity scale. Many athletes have minor symptoms of illness every day which may represent a normal response to high levels of training. The classification could be: 'No' indicating completely asymptomatic or soreness expected from training and not unusual for the athlete, 'Low' indicating mild discomfort during exercise or training, but not impinging on duration or intensity, for example; 'Moderate' as limiting training; and 'High' leading to inability to train. 2) Illness: many subjects found 'illness' easier to classify using Yes/No criteria, but thought it might be helpful to note when an athlete is feeling 'on edge' or feeling as if they are fighting off being ill. 3) Symptom categories: the category 'Other (please specify)', might include 'stress', which is quite different from 'Psychological (depression and anxiety)', and may be due to travel, for example, which can be a stressor since many athletes undertake extensive domestic and international travel.

\section{Discussion}

This study involved the development, administration, and evaluation of a self-reported illness questionnaire for athletes. Feedback provided by sports physicians and medical experts on the previous version of the athletes' self-reported illness questionnaire facilitated the development of the revised questionnaire. The questionnaire included the essential symptoms athletes are likely to experience and had clearer instructions to enhance usability. Substantial differences in illness duration and illness load were identified between highly-trained runners and age- and sex-matched recreationally-active individuals. This research has value for sport scientists, and athletes and their coaches in particular, who need to identify illness in order to modify training and competition schedules to minimize the severity of illness and promote recovery.

Previous research examined the impact of illness on athlete performance, measured by treadmill testing, and included 
Table 3 Distribution of illness symptoms in highly-trained runners and control subjects

\begin{tabular}{|c|c|c|c|c|c|}
\hline \multicolumn{3}{|l|}{ Controls } & \multicolumn{3}{|l|}{ Runners } \\
\hline Symptom & Frequency & Ranking & Symptom & Frequency & Ranking \\
\hline URTI & 19 & 1 & Fatigue & 16 & 1 \\
\hline Head & 17 & 2 & URTI & 10 & 2 \\
\hline Fatigue & 16 & 2 & Head & 8 & 3 \\
\hline GI & 11 & 4 & $M \& J$ & 7 & 4 \\
\hline M\&J & 8 & 5 & Psychol & 7 & 4 \\
\hline Eye & 8 & 5 & GI & 6 & 6 \\
\hline Chest & 5 & 7 & Other & 4 & 7 \\
\hline Skin & 5 & 7 & Chest & 3 & 8 \\
\hline Psychol & 4 & 8 & Eye & 2 & 9 \\
\hline Ears & 2 & 9 & Skin & 2 & 9 \\
\hline Other & 1 & 10 & $C V$ & 1 & 11 \\
\hline Rashes & 0 & 11 & Rashes & 0 & 12 \\
\hline$C V$ & 0 & 11 & Ears & 0 & 12 \\
\hline Urinary & 0 & 11 & Urinary & 0 & 12 \\
\hline
\end{tabular}

Abbreviations: URTI, upper respiratory tract illness; GI, gastrointestinal; M\&J, muscle and joints; Psychol, psychological; CV, cardiovascular.

symptoms verified by a physician. ${ }^{3}$ In our study, we sought to create a daily self-reported illness questionnaire that, without physician review, is useful in characterizing patterns of illness and their impact on athletes' training, and allows an analysis of illness and training loads. In addition, we used a composite illness load, calculated by the addition of daily illness severity (1-3) for each illness episode, in contrast to that in our earlier study (duration $\times$ peak severity). ${ }^{3}$ For these reasons we consider our current method gives a more accurate representation of the illness episode.

Athletes who filled in the questionnaire reported that it took no more than five minutes a day and was clear and easy to follow. One hundred percent of athletes participating in the study over the three month period completed their illness $\operatorname{logs}$ each day, indicating that the questionnaire met the aims of practicality and conciseness. The distinctive aspect of our self-reported illness questionnaire was incorporation of a 'functional' element in the reporting of illness severity. This metric was determined by the degree to which subjects were impeded in carrying out specified activities (ie, training sessions for athletes and general physical activity for medical students) and relating this to symptoms. Clear explanation of this feature of the illness questionnaire to participants was crucial for accurate symptom reporting and objective self-reported symptoms.

The training load (the product of training duration and intensity) undertaken by elite athletes was an important parameter, owing to its potential to contribute to athlete health or illness. Earlier studies proposed moderate exercise has the potential to boost immune function and that aerobic training may have an important role in maintaining immune competence in highly-trained athletes. ${ }^{14}$ In this way regular exercise training can provide a protective buffer during more intensive specific training and competition phases..$^{14}$ An explanation for our findings may be that athletes exercising at high intensities do not always allow sufficient time to recover between sessions, contributing to the onset of fatigue and immune system compromise. This assertion is supported by the notion that, in highly-trained athletes, an imbalance between training loads and recovery due to overtraining, or sudden increases in either training volume or intensity, or a combination of these, may place additional pressure on immune function. ${ }^{14}$ We recommend that athletes, together with their coaches, use a self-reported questionnaire to monitor illness symptoms and identify training patterns that minimize illness and maintain health and performance.

In addition to excessive physical stress (high training workloads - frequency, duration, volume, and intensity), the longer duration of illness and greater illness load of highlytrained athletes may be a product of psychological stress (eg, sport-specific anxiety). Our runners were undertaking summer training leading to the national championships. Increased exposure to pathogens in the training or competition environment from team mates, opponents and team officials, and environmental conditions (hot or cold temperatures, polluted air, and high altitudes) may trigger hyperventilation, irritation and drying of airways, ${ }^{18}$ contributing to increased symptom reporting. Elite distance runners may experience a 
greater illness load and duration of illness and presentation of upper respiratory symptoms, owing to the nature of the sport which involves prolonged and intensive exercise and passage of large volumes of air through the respiratory tract. This may lead to mucosal drying and transient immunosuppression, or physical damage to the mucosa leading to a subsequent inflammatory response. These effects may be perceived by athletes as symptomatic of an upper respiratory tract illness (URTI). ${ }^{1}$ A future study investigating illness patterns and training loads in highly-trained athletes participating in other sports (eg, team sports such as netball and football) would provide a useful comparison with our research.

For athletes and coaches seeking to minimize the impact of illness on training and exercise performance, these findings emphasize the importance of early detection of illness in athletes and the value of modifying training programs in order to minimize the severity and duration of illness. ${ }^{19}$ This information is vital as the persistence of strenuous training during times of illness can have deleterious physical effects on athletes. Intense exercise during the acute phase of a viral illness may increase the severity and duration of the illness with serious consequences..$^{20}$ Rarely, more serious consequences, such as sudden death from myocarditis due to viral infection can occur. ${ }^{21}$ An athlete experiencing 'systemic' symptoms (eg, fever, fatigue, elevated heart rate, myalgia, arthralgia, and lymphadenopathy), which indicate a more significant illness, has a greater need to rest from competition and training. ${ }^{21}$ Our self-reported illness questionnaire can assist athletes and their coaches by drawing attention to systemic symptoms, and helping them modify training programs to minimize the impact on health, training, and athletic performance.

Self-reported illness questionnaires have a number of commonly recognized limitations that reduce their usefulness in a research or clinical setting. In particular, reports of symptom measures reflect subjectively experienced well-being rather than objective health status (ie, symptom reports do not necessarily reflect illness). ${ }^{7}$ There is also room for under-reporting as it relies on the recall of subjects who may have trouble understanding the terms used in the questionnaire. ${ }^{22}$ The difficulty in recruiting a representative sample, variability in symptom perception, and difficulty in interpreting severity scales (not tied to universally understood reference standards) all reduce the usefulness of self-reported illness logs. ${ }^{9}$ Another difficulty with self-reported illness is that, initially, respondents may become increasingly sensitive to and aware of their physical and mental status (and thus increase symptom reporting), but over time may become tired of completing the same questions daily (and therefore reduce symptom reporting and provide less accurate records). ${ }^{7}$ Athletes commonly experience musculoskeletal injury and pain, ${ }^{23}$ and it is desirable for athletes and clinicians to differentiate these symptoms from the onset of illness. We developed our self-reported illness questionnaire in light of these limitations, with the goal of improving a number of aspects to enhance the usefulness in an elite athlete population.

\section{Conclusions}

The athlete illness log is useful for quantifying the pattern of self-reported symptoms of illness in field settings. Despite the limitations of self-reported illness questionnaires, a well constructed, concise questionnaire that focuses on the extent to which athletes record impacts on training associated with their symptoms is of practical benefit for athletes and coaches seeking to minimize the impact of illness on health, training, and exercise performance. Highly-trained runners experience substantially longer episodes of illness (mean of $\sim 6$ days of symptoms) with a greater impact on daily activity than recreationally-active individuals ( $\sim 3$ days of symptoms). Analysis of self-reported symptoms of illness could be a useful adjunct (particularly as a point-of-care screening process) to traditional clinical and laboratory evaluation of respiratory symptoms in elite athletes.

\section{Acknowledgements}

The involvement and cooperation of the medical staff and subjects through the Australian Institute of Sport and its network is gratefully acknowledged. The contribution of academic staff and students of the Australian National University Medical School is also acknowledged.

\section{Disclosures}

The authors report no conflicts of interest in this work.

\section{References}

1. Cox AJ, Gleeson M, Pyne DB, Saunders PU, Clancy RL, Fricker PA. Valtrex therapy for Epstein-Barr virus reactivation and upper respiratory symptoms in elite runners. Med Sci Sports Exerc. 2004;36:1104-1110

2. Gleeson M, Pyne DB. Respiratory infections and mucosal immunity in athletes. Am J Med Sport. 2001;3:159-165.

3. Fricker PA, Pyne DB, Saunders PU, Cox AJ, Gleeson M, Telford RD. Influence of training loads on patterns of illness in elite distance runners. Clin J Sports Med. 2005;15:244-250.

4. Pyne DB, Gleeson M, Fricker PA, Batterham A, Hopkins WG. Characterising the individual performance responses to mild illness in international swimmers. Brit J Sports Med. 2005;39:752-756.

5. Maille AR, Koning CJ, Zinderman AH, Willems LN, Dijkman KH, Kaptein AA. The development of the 'Quality-of-life- for Respiratory Illness Questionnaire (QOL-RIQ): a disease-specific quality-of-life questionnaire for patients with mild to moderate chronic non-specific lung disease. Resp Med. 1997;91:297-309. 
6. Cox AJ, Gleeson M, Pyne DB, Callister R, Hopkins WG, Fricker PA. Clinical and laboratory evaluation of upper respiratory symptoms in elite athletes. Clin J Sports Med. 2008;18:438-445.

7. Gijsbers van Wijk CMT, Huisman H, Kolk AM. Gender differences in physical symptoms and illness behaviour: a health diary study. Social Sci Med. 1999;49:1061-1074.

8. Currie A, Potts SG, Donovan W, Blackwood D. Illness behaviour in elite middle and long distance runners. Brit J Sports Med. 1999;33:19-21.

9. Barrett B, Brown R, Mundt M, et al. The Wisconsin Upper Respiratory Symptom Survey is responsive, reliable, and valid. J Clin Epidemiol. 2005;58:609-617.

10. Barrett B, Brown R, Mundt M. Comparison of anchor-based and distributional approaches in estimating important difference in common cold. Qual Life Res. 2008;17:75-85.

11. Gvozdenovic BS, Mihailovic-Vucinic V, Ilic-Dudvarski A, Zugic V, Judson MA. Differences in symptoms severity and health status between patients with pulmonary and pulmonary plus extrapulmonary sarcoidosis. Resp Med. 2008;102:1636-1642.

12. Tiollier E, Gomez-Merino D, Burnat P, Jouanin JC. Intense training: mucosal immunity and incidence of respiratory infections. Eur J Appl Physiol. 2005;93:421-428.

13. Smith JA, Pyne DB. Exercise, training, and neutrophil function. Exer Immunol Rev. 1997;3:96-116.

14. Pyne DB, Gleeson M, McDonald WA, Clancy RL, Perry CP, Fricker PA. Training strategies to maintain immunocompetence in athletes. Int $J$ Sports Med. 2000;21:S51-S60.
15. Sellar CM, Syrotuik DG, Field CJ, Bell GJ. The effect of dietary control and carbohydrate supplementation on the immune and hormonal responses to rowing exercise. Appl Physiol Nutr Metab. 2006;31:588-596.

16. Neville V, Gleeson M, Folland JP. Salivary IgA as a risk factor for upper respiratory infections in elite professional athletes. Med Sci Sports Exerc. 2008;40:1228-1236.

17. Batterham AM, Hopkins WG. Making meaningful inferences about magnitudes. Int J Sport Physiol Perform. 2006;1:50-57.

18. Fricker PA, Pyne DB. Why do athletes seem prone to infection? Med Today. 2005;6:66-67.

19. Anderson L, Triplett-McBride T. Impact of training patterns on incidence of illness and injury during a women's collegiate basketball season. J Strength Cond Res. 2003;17:738-744.

20. Brennan F, Stenzler B, Oriscello R. Diagnosis and management of myocarditis in athletes. Curr Sports Med Rep. 2003;2:65-71.

21. Friman G, Wesslen L. Special feature for the Olympics: effects of exercise on the immune system: infections and exercise in high-performance athletes. Immunol Cell Biol. 2000;78:510-522.

22. Fricker PA, Gleeson M, Pyne DB, Flanagan AJ, McDonald WA, Clancy RL. A clinical snapshot: do elite swimmers experience more upper respiratory illness than non-athletes? Clin Exerc Physiol. 2000;2:155-158.

23. Anglem N, Lucas SJ, Rose EA, Cotter JD. Mood, illness and injury responses and recovery with adventure racing. Wild Environ Med. 2008;19:31-38.
Open Access Journal of Sports Medicine

\section{Publish your work in this journal}

Open Access Journal of Sports Medicine is an international, peer-reviewed, open access journal publishing original research, reports, reviews and commentaries on all areas of sports medicine. The manuscript management system is completely online and includes a very quick and fair peer-review system.

\section{Dovepress}

Visit http://www.dovepress.com/testimonials.php to read real quotes from published authors. 